\section{links of Iron}

PeruTz's haemoglobin crystallography turns out to be mightily powerful juju, for not only has he now established the precise relation between the coordination state of the haem iron, the structural changes within the subunits, their disposition relative to each other, and the affinity of the molecule for its ligands, but he has also found answers to questions that have exercised inorganic chemists and spectroscopists since the days of the biochemical ice age. Two articles, one by Perutz on page 495 of this issue of Nature, and one by Perutz, Pulsinelli and Ranney in this week's Nature New Biology (237, 259 ; 1972), tell the story.

Some important pointers to the truth emerged from the structural properties of an abnormal human haemoglobin, known as M Milwaukee. The designation haemoglobin $\mathbf{M}$ implies that, as it comes out of the patient, the pigment contains ferric haems, which are unable to bind oxygen. In general this is a consequence of the substitution of tyrosine for histidine as a ligand for the iron. In the Milwaukee variant, however, the mutation, which occurs in the $\beta$ chains, consists of the replacement of a valine by glutamic acid in the haem pocket, on the oxygen-binding side of the haem plane. From difference Fourier maps it can be deduced that the carboxylate group of the glutamic acid is a haem ligand, which interacts strongly with the ferric ion by virtue of its charge.

A more remarkable result, however, is that the crystal structure of deoxyhaemoglobin $\mathbf{M}$ Milwaukee is isomorphous with that of normal deoxyhaemoglobin, rather than oxy- or methaemoglobin, as one would expect on the basis of experience with other haemoglobin $M$ variants, as well as artificial hybrids of deoxygenated $\alpha$ chains, and cyanomet $\beta$ chains. When the $\alpha$ chains are in the oxygenated state, however, the crystals become isomorphous with those of normal oxy- (or met-) haemoglobin. If now the deoxygenated form of the Milwaukee variant is in the characteristic deoxyhaemoglobin state of quaternary structure (in allosteric vernacular, the $T$ state), one may surmise that the ferric subunits are in the corresponding conformational state (which Perutz designates $t$ ); but when the $\alpha$ chains are oxygenated, the sequence of events previously enumerated by Perutz-starting with the shift of the iron atom into the haem plane, which pulls its histidine ligand with it, and activates the system of rods, pulleys, cogs and levers that finally breaks the critical inter-chain salt links-is set in train. The consequence would be that the $\beta$ chains would enter their other conformational state $(r)$, and the tetramer as a whole its $R$ structure.

The implication is that the iron atom in the ferric subunits can be made to move into or out of the haem plane according to the demands of the two conformations. This displacement should in turn be manifested in changes of the spin state of the iron, which is governed by the haem geometry, and consequently, if theoretical divinations are correct, of certain features in the visible absorption spectrum. In particular, bands at 630 and $1,000 \mathrm{~nm}$ have been identified with the low-spin condition, and when for example methaemoglobin, which in the acid form has water in the sixth coordination position, is titrated through its spectroscopic $p \mathrm{~K}$ to the high-spin hydroxyl form, the band shifts and fades in intensity. In deoxyhaemoglobin M Milwaukee the ferric chains do not give rise to either of these low-spin absorption bands. When, however. the $\alpha$ chains are liganded with carbon monoxide, the characteristic bands-due to the ferric $\beta$ chains-appear. Thus a primary event at the $\alpha$ chain haems generates a spectroscopic and structural change in the $\beta$ chains.

Furthermore, in the deoxygenated haemoglobin, the ferric $\beta$ chains, being in the $T$ state, are able to bind the cofactors, diphosphoglycerate (DPG) and inositol hexaphosphate (IHP). In binding, these stabilize the $T$ structure, so that they are able to inhibit the above-mentioned spectral change. It thus seems that the "allosteric constant" $(L)$, which is simply the equilibrium constant for the $T-R$ transition, has an anomalous value in the Milwaukee variant. Other properties, such as the presence of a Bohr effect, can then be readily rationalized.

Perutz descants on the same theme in considering haemhaem interactions in general. He has shown by examination of the absorption spectra of ferrous-ferric hybrids of normal haemoglobin in the spin-sensitive regions that here too the ferric $\beta$ chain responds to the ligation state of the ferrous $\alpha$ chain, and that the $T-R$ equilibrium can be controlled by addition or removal of the cofactors. There is thus a reciprocal reaction or, as Perutz has it, a seesaw, involving on the one hand the balance of the high spin and low spin states of the pigment (manifestations of the position of the iron relative to the haem plant), and on the other that of the $R$ and $T$ conformations. The same reasoning holds also for the wholly ferrous state: in the $T$ geometry (that of deoxyhaemoglobin) the constraints of conformation impose a large out-of-plane displacement of the iron, which inhibits the oxygenation reaction, and is thus responsible for the low oxygen affinity of the deoxyhaemoglobin.

An interesting byproduct of all this is an explanation for the hitherto somewhat supererogatory observations on hyperfine shifts in the haemoglobin proton magnetic resonance (PMR) spectrum. The phenomena that are observed during oxygenation may now be seen in terms of the change in the spin state of the iron, which governs the magnitude of the contact-shifts. Close examination of the shift profiles shows that they represent a two-state process. Moreover, the observations on ferrous-ferric hybrids, in which oxygenation of $\alpha$ chains affects the magnitudes of chemical shifts in the resonances arising from the $\beta$ chains, also now become comprehensible. Moreover, in this week's Nature New Biology (237, 263; 1972) Lindstrom, Ho and Pisciotta report that the oxygenation of the $\alpha$ chains in haemoglobin M Milwaukee leads, as one is now entitled to expect, to marked changes in the features of the hyperfine-shifted PMR spectrum assigned to the ferric $\beta$ chains. The manner in which such diverse observations now fall into place is one of the most pleasing aspects of Perutz's new theory.-From our Molecular Biology Correspondent. 